\title{
Optimization design of optical waveguide control by nanoslit-enhanced $\mathrm{THz}$ field
}

\author{
A. Novitsky, R. Malureanu, M. Zalkovskij, P. Uhd Jepsen, A.V. Lavrinenko \\ DTU Fotonik, Department of Photonics Engineering, Technical University of Denmark, Ørsteds pl. 343, DK-2800 Kgs. Lyngby, Denmark
}

Received 29 April 2013; received in revised form 6 September 2013; accepted 10 September 2013

Available online 2 October 2013

\begin{abstract}
We discuss design issues of devices which were proposed recently [Opt. Lett. 37 (2012) 3903] for terahertz (THz) control of the propagation of an optical waveguide mode. The mode propagates through a nonlinear dielectric material placed in a metallic nanoslit illuminated by $\mathrm{THz}$ radiation. The $\mathrm{THz}$ field in the slit is strongly localized and thus significantly enhanced, facilitating nonlinear interactions with the dielectric waveguide material. This enhancement can lead to notable changes in the refractive index of the waveguide. The closer the waveguide is to the slit walls, the higher the nonlinear effects are, but with the cost of increasing propagation losses due to parasitic coupling to surface plasmon polaritons at the metal interfaces. We analyze several optical waveguide configurations and define a figure of merit that allows us to design the optimal configuration. We find that designs with less overlap of the THz and optical fields but also with lower losses are better than designs where both these parameters are higher. The estimated terahertz field incident onto the metallic nanoslit required to manipulate the waveguide mode has reasonable values which can be achieved in practice.
\end{abstract}

(C) 2013 Elsevier B.V. All rights reserved.

Keywords: Nanoslit; Terahertz radiation; Field enhancement; Nonlinearity

\section{Introduction}

Terahertz (THz) physics is advancing with a rapid pace [1-5]. One of the potential applications of general interest is the use of the high bandwidth available at $\mathrm{THz}$ frequencies for ultrafast wireless communications. For communication applications the $\mathrm{THz}$ range is unique because it is possible to exploit the benefits of speed and bandwidth allowed by photonic technology for modulation and processing of free-space signals. Efficient all-optical conversion of information between these regions is important in order to be able to interface between wireless and fiber-based technologies in a

E-mail address: alav@fotonik.dtu.dk. larger communication system. This conversion process still is a major technological challenge, firstly due to the large wavelength difference (a factor of 200 between $1 \mathrm{THz}$ and $1.55 \mu \mathrm{m}$ ) which makes even unity photon conversion efficiency very inefficient in terms of power, secondly due to the hampering requirement of a high $\mathrm{THz}$ field strength for nonlinear frequency conversion which is difficult to obtain at long wavelengths due to poor focusability, and thirdly due to a lack of knowledge about fundamental nonlinear properties in the $\mathrm{THz}$ range of most materials.

One of the commonly accepted proposals that address some of these challenges in the $\mathrm{THz}$ range is to apply the appealing properties of metamaterials for field enhancement and localization. Metamaterials, as artificial composite structures having no analogues in 
nature, indeed enable certain solutions, for example, introducing terahertz magnetic response [6] and ultrahigh refractive index designs [7], thereby facilitating applications for modulation and sensing [8-12].

$\mathrm{THz}$ metamaterials have been shown recently to be configured for effective optical modulation (see Ref. [13] and references therein). Such modulation is based on changes of the substrate conductivity induced by photoexcited charge carriers. The optically induced properties observed in the $\mathrm{THz}$ range are comparably easy to induce with state-of-the-art near-infrared femtosecond laser technology. The exploitation of nonlinear phenomena induced by $\mathrm{THz}$ waves in such structures, on the other hand, is still in its adolescence. Most of the results have been reported so far regards nonlinear effects due to quasi-free electrons in semiconductors [14-22], and phonon dynamics in molecular crystals [23]. Meanwhile, it will be intriguing to demonstrate in a direct way the THz-field induced manipulation of the optical properties of transparent optical materials, as employed in conventional, integrated optical waveguides.

In this article we perform a detailed analysis of the design issues of a terahertz modulator of near-infrared waves in an optical waveguide reported recently [24]. The key part of the device functionality relies on a nanoslit enhancement of the terahertz field [25] which can enhance the cross-phase modulation (XPM) between the $\mathrm{THz}$ wave and the optical mode in the waveguide significantly. The nanoslit enhancement has no resonant features such as the enhanced optical transmission [26], but rather a broadband enhancement inversely proportional to frequency. The electric field enhancement arises due to the displacement of electric charges at the edges of the nanoslit. The metal film must be thin enough (sub-skin depth) to ensure that the electrical field in the film drives electrons efficiently [25,27-30]. Since the nanoslit can be thousands of times smaller than the $\mathrm{THz}$ wavelength thus reaching dimensions comparable to the optical wavelength range, it is the connecting element between the two regimes.

Due to the limited knowledge about the magnitude of nonlinearities in the $\mathrm{THz}$ range, deciding what material to use is not a straightforward task. Second-order electro-optic techniques are widely employed for nonlinear generation (optical rectification or difference-frequency generation) and detection ( $\mathrm{THz}$ Pockels effect) of coherent $\mathrm{THz}$ pulses of known polarization and with synchronized arrival at the detector crystal. However, the encoding of arbitrary polarized $\mathrm{THz}$ fields without a known synchronization onto an optical signal is a highly demanding task. Due to the amorphous structure of soft glasses and most other optical waveguide materials, we assume that the second-order nonlinearity of the material is insignificant, and therefore that the nonlinear refractive index change is induced by XPM due to the THz Kerr effect.

Results of recent THz-induced Kerr effect investigations have shown that the magnitude of THz-induced and optically induced Kerr coefficients are similar [31]. We therefore assume that the nonlinear coefficients in the $\mathrm{THz}$ range are of the same order of magnitude as in the optical range. Chalcogenide glasses, and in particular arsenic trisulphide $\left(\mathrm{As}_{2} \mathrm{~S}_{3}\right)$, seem to be suitable candidates as they provide a very high nonlinear coefficient, reasonable low losses, and good confinement of the optical wave due to the high refractive index [32-34]. The linear refractive index of $\mathrm{As}_{2} \mathrm{~S}_{3}$ is known both in the low-terahertz regime (0.1$2 \mathrm{THz})[35,36]$ and at higher frequencies $(2-18 \mathrm{THz})$ as well [37].

The paper is structured as follows. Temporal dynamics with continuous waves (CW) and pulsed fields is discussed in Section 2. The nonlinear response of chalcogenide glass is discussed in Section 3. In Section 4 we analyze several designs inspecting theoretical and experimental advantages and drawbacks of each. First we calculate the distribution of the terahertz field and estimate the nonlinearities in chalcogenide glass in Section 5. In Section 6 we find the characteristics of the optical modes such as propagation constants and losses in the nonlinear waveguide. In Section 7 the field distributions and figures of merit are compared for different designs. Section 8 concludes the paper.

\section{Temporal dynamics}

We can achieve the modulation of an optical signal by using either $\mathrm{CW}$ or pulsed $\mathrm{THz}$ waves, each with advantages and disadvantages. The general configuration of the device is shown in Fig. 1a, which defines three important times, namely the duration of the $\mathrm{THz}$ signal $\tau_{T H z}$, the duration of the optical pulse $\tau_{\text {laser }}$, and the transit time of the optical signal through the interaction region $\tau_{\text {transit }}=n_{W G} L / c$, where $L$ is the length of the active region, $n_{W G}$ is the group index. In the following sections we deal with the monochromatic waves, therefore, $n_{W G}$ is just refractive index.

The quasi-CW regime is when the $\mathrm{THz}$ signal is longer than the transit time of the optical probe, $\tau_{T H z}>\tau_{\text {transit }}$, and the optical probe signal is of longer duration than the duration of the $\mathrm{THz}$ signal 


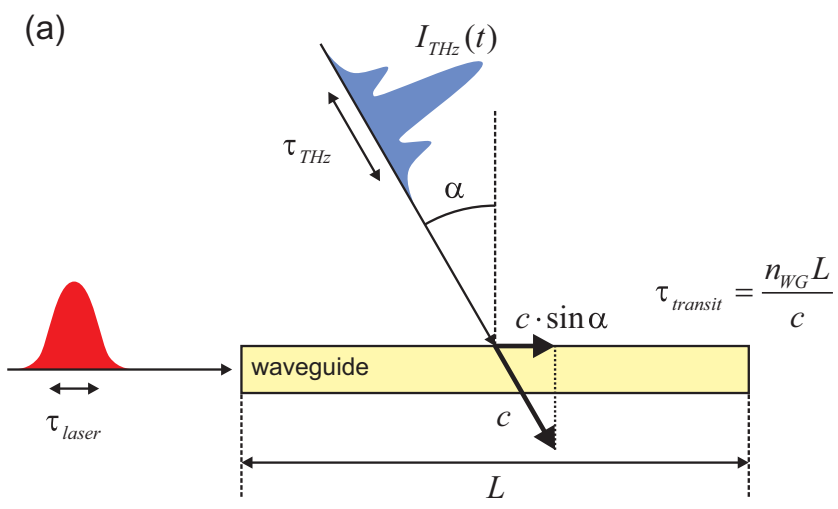

(b)

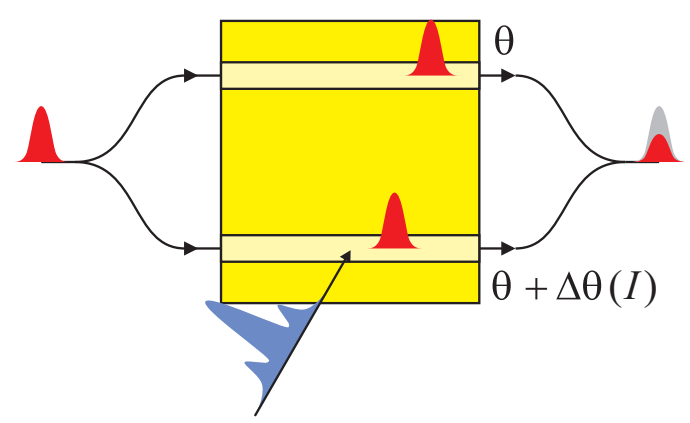

Fig. 1. (a) Schematic illustration of the geometry of interaction between a THz field of duration $\tau_{T H z}$ incident at an angle $\alpha$ and an optical probe signal of duration $\tau_{\text {laser }}$ in a nonlinear waveguide of length $L$. (b) Detection of the THz-induced phase shift of the optical probe in a Mach-Zehnder configuration.

$\left(\tau_{\text {laser }}>\tau_{T H z}\right)$. For a 1-mm device length, this corresponds to $\tau_{T H z} \approx 7 \mathrm{ps}$. In this regime the modulation of the probe beam due to the $\mathrm{THz}$ beam must be read out without optical sampling techniques, and there are no strict requirements on the synchronization between the $\mathrm{THz}$ signal and the optical probe signal.

For shorter THz signals we are in the pulsed regime. Here the complete $\mathrm{THz}$ signal interacts with the optical probe within less than the transit time of the device $\left(\tau_{T H z}<\tau_{\text {transit }}\right)$. If the optical probe pulse is much shorter than the $\mathrm{THz}$ signal then the device works as an optical sampling unit, and the shape $I_{T H z}(\tau)$ can be sampled by gradual variation of the timing between the $\mathrm{THz}$ signal and the optical probe. This is useful in situations where the detailed shape of the $\mathrm{THz}$ signal must be known, and is similar to the conventional electro-optic sampling techniques used for coherent $\mathrm{THz}$ detection [38-40].

If the duration of the optical probe pulse is longer than the duration of the $\mathrm{THz}$ signal $\left(\tau_{\text {laser }}>\tau_{T H z}\right)$ then the interaction effectively imprints the temporal profile of the $\mathrm{THz}$ signal onto the temporal profile of the optical probe signal. This is useful for single-shot detection of $\mathrm{THz}$ signal waveforms in connection with chirped probe signals [41]. In this a scenario, no temporal scanning is required, and only synchronization between the train of $\mathrm{THz}$ signals and the optical probe signal train is required.

The modification of the refractive index $\Delta n$ and thus the nonlinear phase shift $\Delta \Phi$ induced by the $\mathrm{THz}$ field can be detected for instance by a standard MachZehnder interferometric configuration as shown in Fig. 1b, where the phase chance is converted into an intensity modulation of the transmitted optical probe signal due to interference between the phase-modulated signal and a copy of the original probe beam.

Irrespective of the operational conditions discussed above, temporal smearing of the $\mathrm{THz}$ signal with respect to the probe beam can be minimized by optimizing the angle of incidence $\alpha$ of the THz signal onto the device, as shown in Fig. 1a. The THz field and the optical probe field should sweep across the interaction region with the same velocity in order to avoid smearing of the nonlinear response, much akin to the phase matching in other nonlinear frequency conversion processes. The ideal velocity matching can be obtained at oblique incidence of the $\mathrm{THz}$ beam onto the interaction region at angle $\alpha=\arcsin \left(1 / n_{W G}\right)$ 
(Fig. 1a). For optical indices in the vicinity of $n_{W G}=2.6$, the incidence angle should thus be approximately $23^{\circ}$. At normal incidence of the $\mathrm{THz}$ field, significant temporal smearing will occur even for interaction lengths of a few tens of micrometers, thus severely limiting the bandwidth and effectiveness of the device [42].

For the remainder of this paper we will assume that the optical and terahertz waves interact within the whole length of the device by appropriate synchronization of the effective sweep velocities across the device active region.

\section{Nonlinear response}

We consider the chalcogenide glass $\mathrm{As}_{2} \mathrm{~S}_{3}$ as the nonlinear medium. It is well studied in optics, because it can provide an incredibly high nonlinear Kerr coefficient, more than hundred times larger than that of silica [34]. This is very promising for waveguiding [33,43], slow-light applications [44], sensors and demultiplexers $[45,46]$. In this paper, the value of the nonlinear refractive index in optics $n_{2}=1.1 \times 10^{-17} \mathrm{~m}^{2} / \mathrm{W}$ is transferred to the terahertz range. This nontrivial assumption is justified by the fact that the third-order nonlinear terahertz response for a range of liquids is of the same order of magnitude as in the optical range [31]. Of course, for more precise simulations, the correct values of the nonlinear coefficients need to be measured.

In general, the nonlinear polarization induced by the electric field reads

$P_{i}=\varepsilon_{0}\left(\varepsilon^{L}-1\right) E_{i}+\varepsilon_{0} \sum_{j, m, n=1}^{3} \chi_{i j m n}^{(3)} E_{j} E_{m} E_{n}$,

where $\varepsilon_{0}$ is the vacuum dielectric permittivity, $\mathbf{E}=\left(E_{i}\right)$ is the electric field vector, $\varepsilon^{L}$ is the linear permittivity, $\chi_{i j m n}^{(3)}$ is the third-order susceptibility tensor.

The nonlinear modification of the optical wave is found by insertion of the strong terahertz $\mathbf{E}^{T H z}=E^{T H z} \mathbf{e}_{x}$ and orthogonal weak optical $\mathbf{E}^{\text {opt }}=E^{\text {opt }} \mathbf{e}_{y}$ fields in Eq. (1) and leaving only the terms proportional to $\mathbf{E}^{\text {opt }}$ :

$P_{y}^{o p t}=\varepsilon_{0}\left(\varepsilon^{L}-1\right) E_{y}^{o p t}+3 \varepsilon_{0} \chi^{(3)}\left|E_{x}^{T H z}\right|^{2} E_{y}^{o p t}$,

where $\chi^{(3)}=\chi_{y y x x}^{(3)}=\chi_{y x x y}^{(3)}=\chi_{y x y x}^{(3)}$.

Molecules in a chalcogenide glass can be described as a set of intimate valence alteration pairs (IVAPs) of positively and negatively charged atoms. Using this model, the polarization of glass is generated by the dipole moments of the IVAPs $[47,48]$. When the dipoles

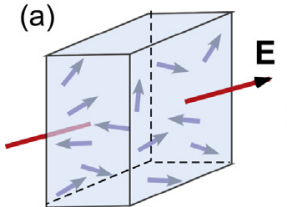

(b)
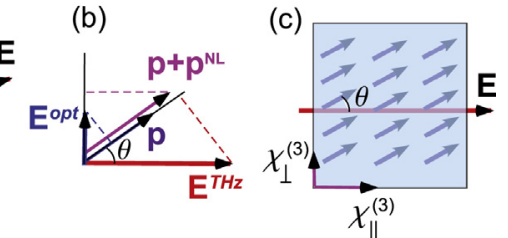

Fig. 2. (a) Randomly oriented IVAP dipoles result in the nonlinearity. (b) Nonlinear molecular polarization $\mathbf{p}^{N L}$ arises owing to the projections of $\mathrm{THz}$ and optical fields onto the dipole direction. This nonlinear polarization vector is then projected onto the direction of the optical field to take into account the nonlinearity of the optical wave induced by the strong $\mathrm{THz}$ field. (c) Oriented dipoles can generate nonlinear response parallel and perpendicular to the electric field.

are randomly oriented (Fig. 2a), the nonlinear response can be calculated by averaging over all directions of the dipoles. If a dipole moment forms an angle $\theta$ with the polarization of the terahertz electric field (Fig. 2b), the polarization of the IVAP changes due to the Kerr effect as

$p_{y}^{N L}=\varepsilon_{0} \gamma\left(E^{T H z} \cos \theta\right)^{2}\left(E^{o p t} \sin \theta\right) \sin \theta$,

where $\gamma$ is the response of a single dipole. The average polarization of the dipoles is then

$$
\begin{aligned}
P_{y}^{N L} & =\left\langle p_{y}^{N L}\right\rangle \\
& =\frac{\varepsilon_{0} \gamma\left|E^{T H z}\right|^{2} E^{o p t}}{4 \pi} \times \int_{0}^{\pi} d \theta \int_{0}^{2 \pi} d \varphi \cos ^{2} \theta \sin ^{3} \theta \\
& =\frac{2}{15} \varepsilon_{0} \gamma\left|E^{T H z}\right|^{2} E^{o p t}
\end{aligned}
$$

for the dipoles in 3D space and

$$
\begin{aligned}
P_{y}^{N L} & =\left\langle p_{y}^{N L}\right\rangle=\frac{\varepsilon_{0} \gamma\left|E^{T H z}\right|^{2} E^{o p t}}{2 \pi} \int_{0}^{\pi} \cos ^{2} \theta \sin ^{2} \theta d \theta \\
& =\frac{1}{16} \varepsilon_{0} \gamma\left|E^{T H z}\right|^{2} E^{o p t}
\end{aligned}
$$

for the in-plane dipoles. Eqs. (4) and (5) model how the nonlinear polarizability stems from the response of randomly distributed elementary dipoles.

Chalcogenide glasses can have an anisotropic distribution of dipole orientation (Fig. 2c). Such orientation can be obtained, e.g., by the LangmuirBlodgett technique [49]. Then the third-order optical nonlinearity also becomes anisotropic. When the dipoles are oriented parallel to the polarization of the 
electric field, the $\chi^{(3)}$ tensor element is 4 times greater than that for perpendicular orientations [50].

As well-known an isotropic nonlinear material has refractive index

$n^{o p t}=n_{0}^{o p t}+\frac{n_{2} \varepsilon_{0} c n_{0}^{o p t}\left|E^{T H z}\right|^{2}}{2}$,

where $n_{2}=3 \chi^{(3)} /\left[2\left(n_{0}^{o p t}\right)^{2} \varepsilon_{0} c\right]$ is the Kerr coefficient and $n_{0}^{\text {opt }}$ is the linear refractive index in the optical range. In general, $n_{0}^{\text {opt }} \neq n_{0}^{T H z}$. In the considered model of the nonlinear medium we neglect the two-photon absorption.

\section{Waveguide designs}

The design configurations we consider can be of three possible types. First, a theory-driven configuration is discussed (Fig. 3a). A gold film of thickness $h$ is deposited on a silica substrate. A chalcogenide glass waveguide is placed in the nanoslit (width $w$ ) etched in the metal. The waveguide has the width $X<w$ and height $Y>X$. In order to obtain efficient guiding there should be a high index contrast between the waveguide and surrounding materials: air and silica. The chalco- genide glass $\mathrm{As}_{2} \mathrm{~S}_{3}$ has high optical refractive index $n_{\text {opt }}=2.3-2.8$ [32] and simultaneously a large Kerr coefficient. The structure (we refer to it as design A) is complex from the point of view of fabrication, but it is preferential as an efficient waveguide providing small losses. The propagation losses are greatly reduced due to the air gaps we introduce between the waveguide and the metal film, thus separating the optical mode from the metal. Large attenuation is caused by the excitation of surface plasmon-polaritons (SPPs) at the edges of the slit, however, this is mainly the case for $x$-polarized optical radiation. If we work with $y$-polarized optical mode (Fig. 3a), the SPP excitation is suppressed, and the waveguide mode is subject to a much lower propagation loss.

The general principle of operation of the device is shown in the inset in Fig. 3a. The terahertz field enhanced by the nanoslit modifies the refractive index of the chalcogenide glass. The optical mode then experiences the variation of the refractive index, thus accumulating the desired phase difference compared to an identical waveguide without terahertz illumination (see Fig. 1). The phase difference can be detected via, for example, a Mach-Zehnder interferometer scheme.
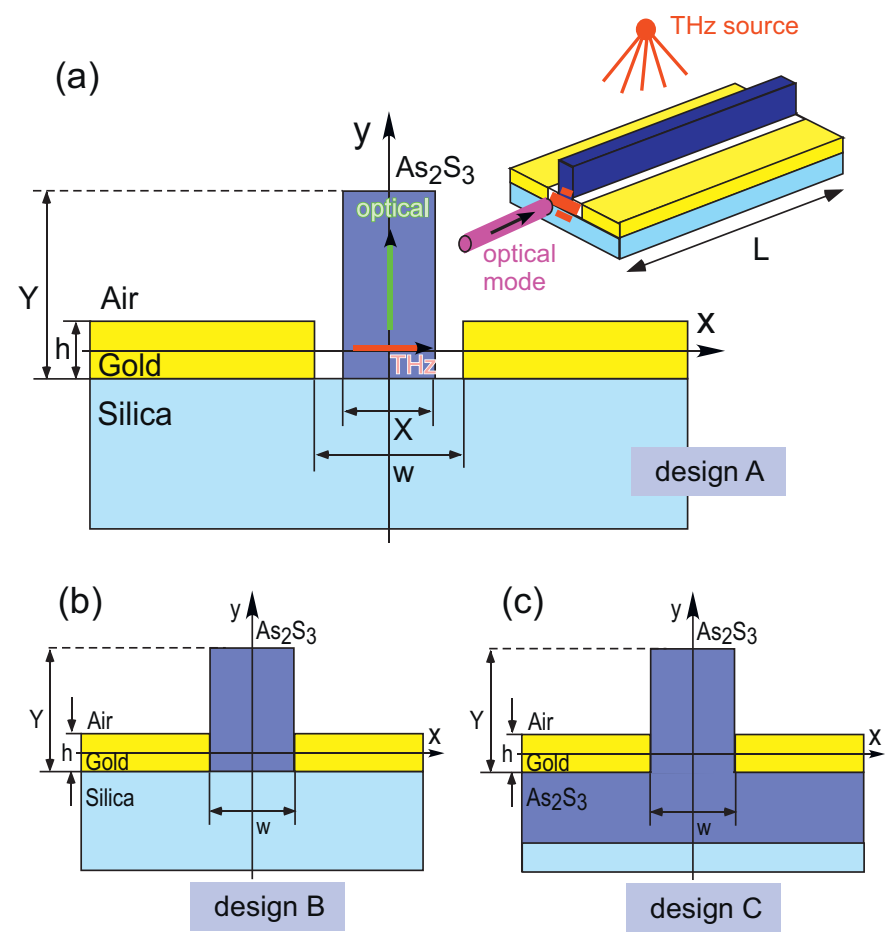

Fig. 3. (a) Theoretically preferable design A with air gaps in the slit. The inset illustrates the terahertz field enhancement in the slit (red spot) and direction of propagation of the optical mode. Designs more preferential for fabrication (b) design B without air gaps and (c) design $\mathrm{C}$ with chalcogenide glass $\mathrm{As}_{3} \mathrm{~S}_{2}$ (dark blue) as a substrate. (For interpretation of the references to color in this figure legend, the reader is referred to the web version of the article.) 
Other designs options we consider have more emphasis on fabrication details. It is obviously easier to fabricate the structure without the air gaps between the metal film and waveguide core - design B (Fig. 3b). Since the mode is close to the metal, propagation losses will be higher for any polarization (while $y$ polarization is still better). It is even easier to fabricate the structure on the chalcogenide substrate - design C (Fig. 3c). In this case, however, the optical mode is less confined in the slit.

\section{THz field distributions}

Using CST Microwave Studio [51], we calculate the field distributions in the nanoslit. Due to the large aspect ratio between the slit dimensions and the $\mathrm{THz}$ wavelength, modeling an isolated slit is extremely challenging. We therefore apply periodic boundary conditions with the distance between the slits of $0.5 \mathrm{~mm}$. A smaller distance would increase the interaction between the slits, thus decreasing the accuracy of the calculations. For any design there are two fields - terahertz and optical - to be studied. They differ widely by frequency, excitation method, and purpose. When calculating the field distributions we assume that the interference between these two fields is negligible. This allows us to calculate the field distributions separately.

Distribution of both terahertz field components $E_{x}$ and $E_{y}$ across the nanoslit is shown in Fig. $4 \mathrm{a}$ and b. The field is enhanced by the electrical charges induced at the slit edges: $E^{s l}=G E^{i n c}+E^{i n c} \approx G E^{i n c}$, where $G$ is the field enhancement factor, and $E^{i n c}$ is the amplitude of the incident field [30]. The $\mathrm{THz}$ field in the nanoslit is similar to the electric field in a plane capacitor being polarized predominantly across the slit in the $x$ direction. Because the slit width is much smaller than the wavelength, the field enhancement is very pronounced. More than one hundred times field enhancement can be easily achieved for the design we consider (Fig. 4a). The $1 / f$ dependence of the field enhancement makes it larger for longer wavelengths.

The field component $E_{y}$ is small compared with $E_{x}$ everywhere except near the four corners of the opening on the metal film, where we also observe strong local field enhancement. Inside the nonlinear waveguide the field $E_{y}$ is very small, especially near the center line $x=0$.

Distribution of the dominating $x$-component of the $\mathrm{THz}$ field in the vertical dimension (along the $y$ coordinate) is shown in Fig. 4c. The maximum of the terahertz field due to the low index silica substrate is
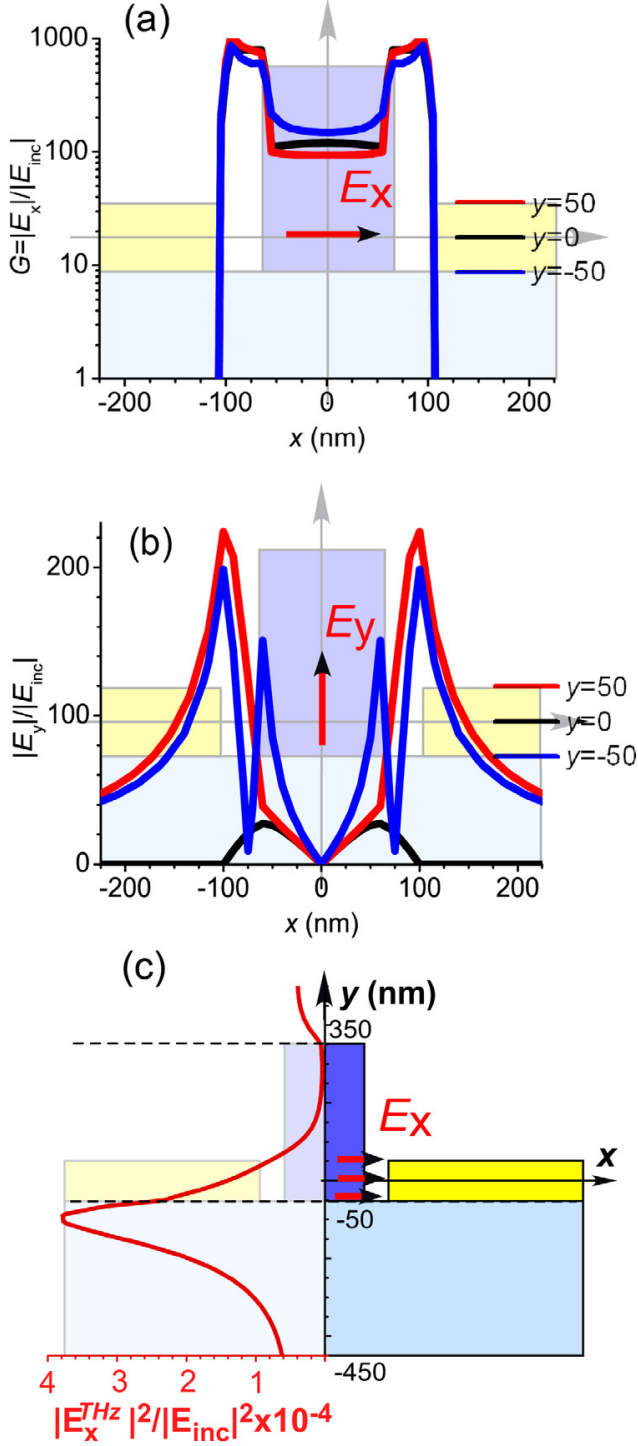

Fig. 4. Field enhancement (a) $G=\left|E_{x}^{T H z}\right| /\left|E_{i n c}^{T H z}\right|$ and (b) $\left|E_{y}^{T H z}\right| /\left|E_{i n c}^{T H z}\right|$ versus coordinate $x$ for $y=-50 \mathrm{~nm}$ (blue curve), $y=0 \mathrm{~nm}$ (black curve), and $y=50 \mathrm{~nm}$ (red curve). (c) Enhancement of the squared $x$-component of the terahertz field as function of coordinate $y$ in cross-section $x=0$. Simulation is performed with parameters $X=120 \mathrm{~nm}, Y=400 \mathrm{~nm}, w=200 \mathrm{~nm}$, and $h=100 \mathrm{~nm}$, $f_{T H z}=0.3 \mathrm{THz}$. (For interpretation of the references to color in this figure legend, the reader is referred to the web version of the article.)

positioned at $y=-50 \mathrm{~nm}$. The field rapidly decays in the chalcogenide glass from $y=-50 \mathrm{~nm}$ to $350 \mathrm{~nm}$. This decrease can be used for a realistic modeling of the change in the refractive index in $\mathrm{As}_{2} \mathrm{~S}_{3}$ (see the following sections).

As we see in Fig. 4a, the THz field is homogeneous in the $x$ direction inside the waveguide. Thus, the refractive index can be modeled in a one-dimensional 
multilayer approach changing properties only along the $y$ axis. The terahertz field enhancement is even larger in the air gaps (see Fig. 4a), but it is outside the nonlinear material and thus, not useful for optical modulation.

We repeat our $\mathrm{THz}$ field analysis for the fabricationoriented designs B and C. The air gaps provide greater field enhancement in the air and shift the terahertz field outside the slit (observe the field maximum in Figs. 4a and $b$ ). Without these air gaps the strong $\mathrm{THz}$ field is screened by the metal film, and the field maximum is in the slit, what is obviously better for the interaction with the waveguide mode.

\section{Optical field distributions}

The optical field is simulated as modes of the ridge chalcogenide waveguide placed in the slit. Such simulations are done in a two-dimensional cross-section geometry, where the waveguide modes at the CST ports are studied. For the optical waveguide modes we determine the propagation constant $\beta$ and field extinction coefficient $\alpha$, which play the main role in the analysis of the THz-optical wave interaction. This aspect is discussed in details in the subsequent Section 7.

In Fig. 5 we show the optical fields of the slab waveguide modes. The distribution of the field in the vertical dimension of design A shows that the field strength is the highest in the center of the chalcogenide waveguide (see Fig. 5a). The mode is well localized inside the dielectric, so only a small fraction of the field reaches the metal film, thus we obtain low propagation losses. In principle the $x$-polarized waveguide mode is also possible, however, in this case, SPPs on the metal interface are effectively excited, so the losses are high. The $y$-polarized mode can be theoretically almost lossless, if the chalcogenide slab is made very tall $(Y \gg X)$. However, such optical mode will overlap only weakly with the terahertz field. This will require an extremely long optical waveguide to accumulate a sufficient nonlinear phase shift. Moreover, for the mechanical stability reasons, the fabrication of a chalcogenide waveguide with a high aspect ratio will be extremely challenging.

In case of configuration B the optical mode is not perfectly $y$-polarized. The $x$-polarized component intensifies the interaction between the optical and $\mathrm{THz}$ waves (due to the 4-times bigger nonlinear constants) but, in the same time reduces the propagation length. The maximum of the optical mode stays at the same position as for design A: near the center of the chalcogenide slab, being insensitive to the width of the slit $w$ (Fig. 5b). When $w=120 \mathrm{~nm}$ the field is forced out of the too narrow waveguide, thereby decreasing the
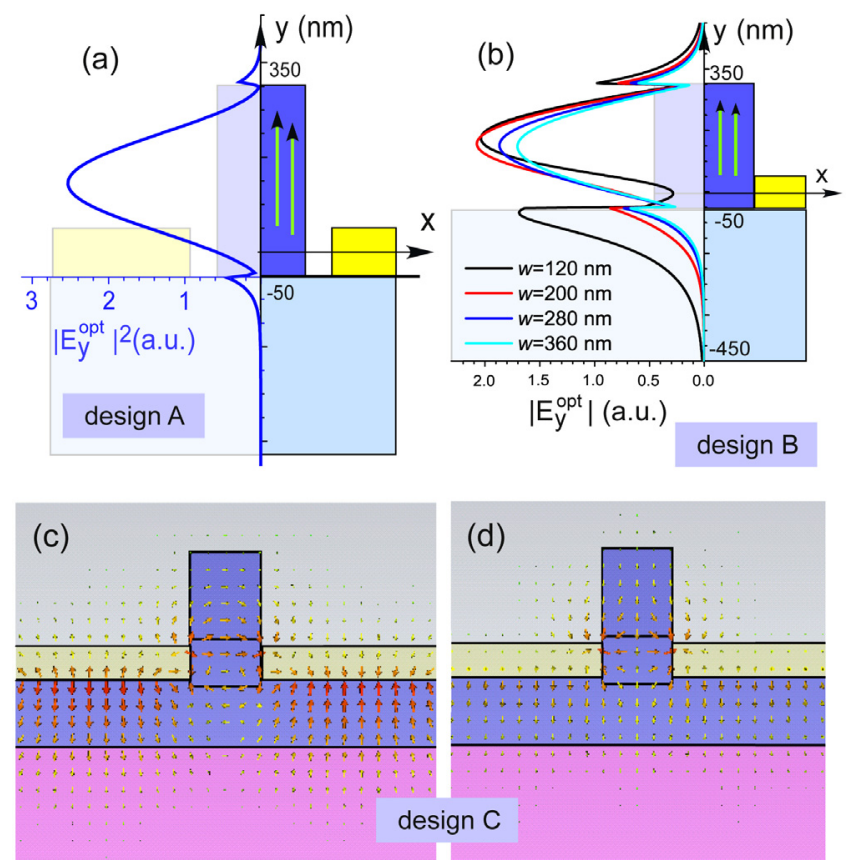

Fig. 5. $y$-Dependence of the squared $y$-electric field of the optical mode calculated using CST Microwave Studio $\left|E_{y}^{\text {opt }}\right|^{2}$. (a) In design A; (b) in design B of different widths. (c) and (d) Field maps of two waveguide modes of design C. If is not shown otherwise parameters are: $X=120 \mathrm{~nm}$, $Y=400 \mathrm{~nm}, w=200 \mathrm{~nm}$, and $h=100 \mathrm{~nm}, \lambda_{\text {opt }}=700 \mathrm{~nm}$. 

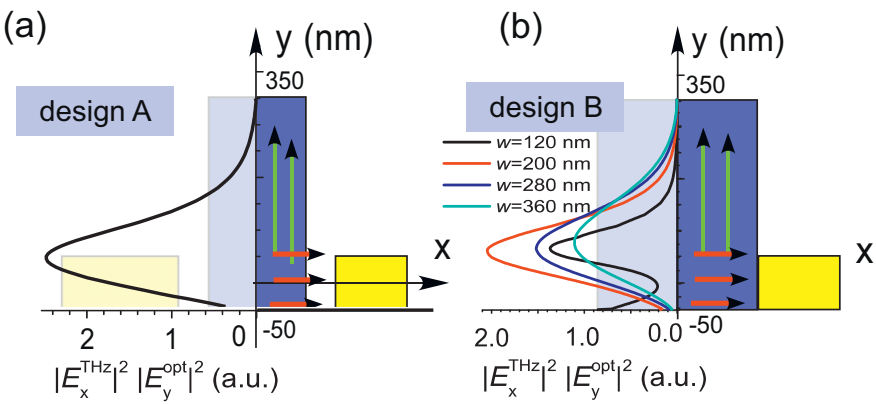

Fig. 6. Overlap function of the optical and THz fields as functions of coordinate $y$. (a) Design A and (b) design B. Parameters: $Y=400 \mathrm{~nm}$ and $h=100 \mathrm{~nm}$.

overlap (interaction) with the $\mathrm{THz}$ field. A wide waveguide will have reduced field intensity. Therefore, the width of the slit thus can be optimized with respect to the interaction strength.

The third design we consider (Fig. 3c) is the easiest to fabricate, but the optical mode is not well localized in the slit anymore. Since there is no index contrast to the substrate, the largest part of the mode is under the metal (Fig. 5c and d). The nonlinear refractive index change takes place near the slit, therefore the total change of the propagation properties of the mode is very small. One more limitation of this design is connected with huge losses in the system, which are expectable, because the optical mode excites SPPs at the metal-substrate interfaces. The structure has too many constraints to compete with the two previous designs (Fig. 3a and b), and we exclude it from the following discussions.

The THz-induced nonlinearity and its influence on the optical mode can be characterized by an interaction function derived from the assumed Kerr nonlinearity of the waveguide material $\Delta n \sim n_{2}\left|E_{x}^{T H z}\right|^{2}$. The coupling of two waves is described as $\int_{\sigma} \Delta n\left|E_{y}^{o p t}\right|^{2} d s$, where $\sigma$ is the area of the waveguide cross-section. Therefore, the interaction function is proportional to the product $\left|E_{x}^{T H z}\right|^{2}\left|E_{y}^{o p t}\right|^{2}$. As we can notice the maxima of the terahertz and optical fields in design A do not coincide. Therefore, the strongest interaction happens between these maxima, namely near $y=h / 2$ (Fig. 6a). Configuration B has the similar trend (Fig. 6b). Variation of the width changes the overlap function (see Fig. 6b). Though the THz field is largest for $w=120 \mathrm{~nm}$, the overlap of the modes is poor. The overlap improves for larger $w$ and then again gets smaller because of the fast decrease of the terahertz field. The biggest overlap is for $w=200 \mathrm{~nm}$, which is the optimal waveguide width for design B.

In the low $\mathrm{THz}$ frequency range (up to $2 \mathrm{THz}$ ), chalcogenide glass is rather low-loss dielectric with refractive index $n_{T H z}=2.7$ [37] (in Ref. [52], $n_{T H z}=2.8$ is mentioned). For higher terahertz frequencies $n_{T H z}$ can vary drastically exhibiting resonance behavior owing to the local vibrational modes of the molecules [37]. In this case the material possesses large losses, e.g. $n_{T H z}=3.2+11.75$ at $f=9 \mathrm{THz}$ and $n_{T H z}=1.8+10.5$ at $f=11 \mathrm{THz}$. However, as we do not expect the $\mathrm{THz}$ field to be guided over long distances in the structure, $\mathrm{THz}$ losses do not play an important role. From the continuity of the electric displacement $\varepsilon E_{x}$ it is evident that the smaller the refractive index, more prominent is the penetration of the electric field into the glass (Fig. 7a). For $n_{T H z}=1.8+10.5$, the electric field is pronounced even for the larger coordinates $y$. However, the enhancement $G$ is very small compared with the enhancement in the low-terahertz range (Fig. 4), because $G \sim 1 / f$. Therefore, there are no obvious benefits in using low refractive indices near $f_{T H z}=10 \mathrm{THz}$.

Although small refractive indices are beneficial in the $\mathrm{THz}$ range, optical waveguide modes are better localized in high-refractive-index materials. In this case, there is a direct connection between the mode localization and mode attenuation: a well localized mode is farther from the metal and, hence, can propagate longer in the chalcogenide waveguide. In Fig. 7b we compare the optical field profiles for two different refractive indices of the glass material: $n_{\text {opt }}=2.7$ (coincides with the refractive index in the THz range) and $n_{\text {opt }}=2.562$ (taken from Ref. [32]). In spite of the mode distribution and hence the overlapping of the $\mathrm{THz}$ and optical waves similarities, the losses in the waveguide mode significantly change from $9 \mathrm{~cm}^{-1}$ (for $n_{\text {opt }}=2.7$ ) to $12.6 \mathrm{~cm}^{-1}$ (for $n_{\text {opt }}=2.562$ ). Thus, the optimal material for the proposed application would be that with a low refractive index in the $\mathrm{THz}$ range and high refractive index in the optical domain. However, such behavior over extended frequency ranges away 

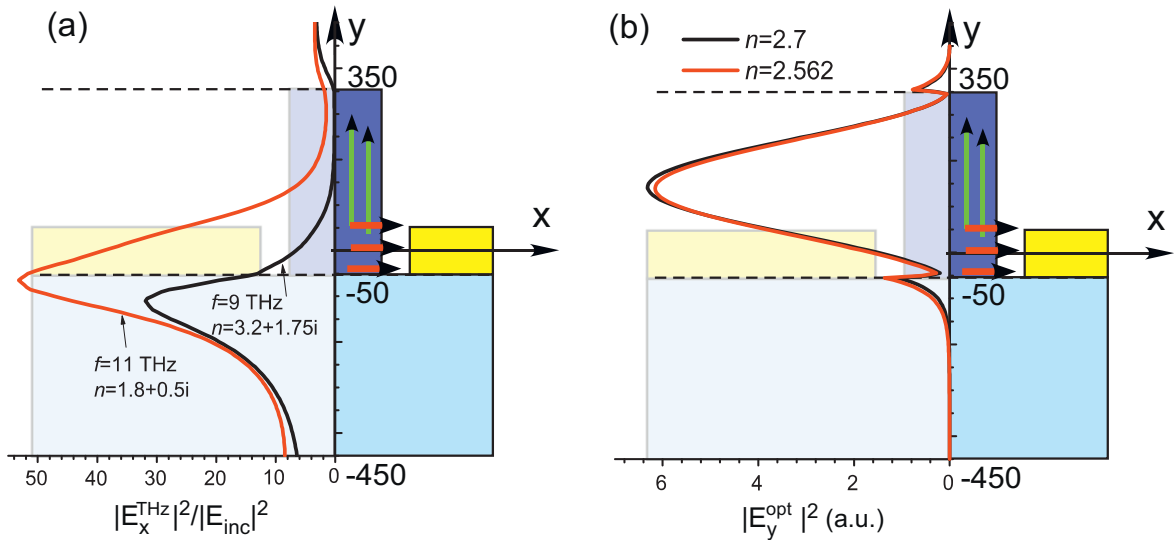

Fig. 7. (a) $\left|E_{x}^{T H z}\right|^{2}$ versus coordinate $y$ for two refractive indices at high terahertz frequencies [37]. (b) $\left|E_{y}^{\text {opt }}\right|^{2}$ versus $y$ at $\lambda_{\text {opt }}=700 \mathrm{~nm}$ for various refractive indices of chalcogenide glass, $n_{\text {opt }}=n_{T H z}=2.7$ and $n_{\text {opt }}=2.562$ as in Ref. [32]. Parameters of design A: $X=120 \mathrm{~nm}, Y=400 \mathrm{~nm}$, $w=200 \mathrm{~nm}$, and $h=100 \mathrm{~nm}$.

from sharp resonances is difficult to obtain with naturally occurring materials.

\section{Characterization and comparison of designs}

As discussed in the previous sections, the THzoptical interaction can be characterized by two quantities, namely (i) the spatial overlap of the $\mathrm{THz}$ and optical fields, and (ii) the propagation length (or attenuation) of the optical mode. A strong overlap leads to a large change $\Delta \beta=\beta-\beta_{0}$ of the modal propagation constant due to nonlinearity, where $\beta$ and $\beta_{0}$ are the propagation constants with and without $\mathrm{THz}$ illumination, respectively. The quantity $\Delta \beta$ helps to simplify description of the $\Delta n$ for modeling purposes. We can approximate the real distribution $\Delta n(y)$ (Fig. 8a) by a step-like function $\Delta n$, where $\Delta n$ is constant within the domain from $y=-h / 2$ to $y=h / 2+\Delta h$ (Fig. 8b) and zero otherwise. As a result of this approximate description the mode profile may differ from the original case with $\Delta n(y)$, but the propagation constant change $\Delta \beta$ is kept the same.

Using the field maps of the THz and optical fields we can evaluate their overlap more precisely. We introduce the normalized dimensionless quantification of the field overlap $\eta$ as

$\eta=\frac{\int_{0}^{Y}\left|E^{T H z}\right|^{2}\left|E^{o p t}\right|^{2} d y}{\left|E_{i n c}^{T H z}\right|^{2} \int_{0}^{Y}\left|E^{o p t}\right|^{2} d y}$.

Generally the integration in the formula should be taken in the whole $(x, y)$ plane. However, the approximately uniform fields distribution in the $x$ - direction reduces the integrals to one-dimensional ones, namely over the $y$ coordinate $(x=0)$. The terahertz field in $\eta$ includes the field enhancement factor $G$, which applies mostly in the slit region. When varying the slit size, two effects must be considered. Firstly, the $\mathrm{THz}$ field enhancement increases when the slit size decreases, leading to an increase in $\eta$. Secondly, outside the slit region the $\mathrm{THz}$ field decreases, and thereby $\eta$ is lowered. Due to this, the integrals should be calculated for the whole height of the structure.

The attenuation of the optical mode defines the interaction length. If losses are large, the propagation distance $D$ is short and the optical mode is not influenced by THz-induced changes to the material properties. For small losses, the optical mode propagates within the nonlinear slab over long distances and the phase shift can be accumulated.

A figure of merit (FOM) is usually introduced for convenience to compare structures which differ in their dimensions or designs (see Fig. 3). The FOM in our case is defined as the ratio of two distances. The first distance is defined as the one the optical wave has to travel in order to achieve an extra $\pi / 2$ phase shift due to the nonlinear interaction. It is thus defined as $L_{\pi / 2}=\pi /$ $(2 \Delta \beta)$ and should be as short as possible. The second distance is the propagation length $D$, the distance where the intensity of the optical mode has been reduced by a characteristic factor of $e, D=1 /(2 \alpha)$.

Thus, we can define the dimensionless FOM as follows [24],

$\mathrm{FOM}=\frac{L_{\pi / 2}}{D}=\frac{\pi \alpha}{\Delta \beta}$, 


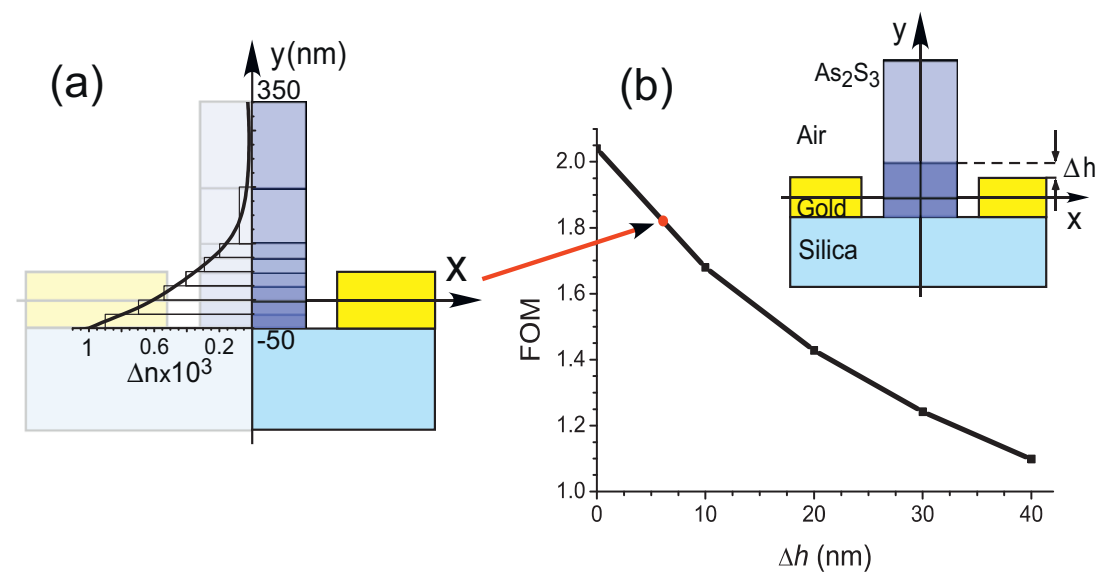

Fig. 8. (a) $y$-Distribution of the nonlinear refractive index change in the optical waveguide and its staircase approximation. (b) Figure of merit (FOM) versus different sizes of the nonlinear region modeled as constant change of the refractive index $\Delta n=0.001$ in the region from $y=-h / 2$ to $y=h / 2+\Delta h$. Red point indicates the FOM for the $y$-dependent refractive index change $\Delta n$ shown in the left panel. Parameters: $Y=400 \mathrm{~nm}$, $X=120 \mathrm{~nm}, h=100 \mathrm{~nm}, w=200 \mathrm{~nm}, f_{T H z}=0.3 \mathrm{THz}$, and $\lambda_{\text {opt }}=700 \mathrm{~nm}$. (For interpretation of the references to color in this figure legend, the reader is referred to the web version of the article.)

with the consequence that the smaller the FOM, the better the performance of the structure.

The FOM for the design with the air gaps between chalcogenide glass and metal is shown in Fig. $8 \mathrm{~b}$. The region of the refractive index change 0.001 is determined by the height $h / 2+\Delta h$ (see the inset in Fig. 8b). When the nonlinear region expands $(\Delta h$ increases), the overlap of the terahertz and optical mode is better, $\Delta \beta$ raises and FOM gets smaller. It means that the system behavior gets better.

We have modeled the design A presented in Fig. 3a with the following parameters: $Y=400 \mathrm{~nm}$, $X=120 \mathrm{~nm}, h=100 \mathrm{~nm}, w=200 \mathrm{~nm}, \lambda_{\text {opt }}=700 \mathrm{~nm}$. Considering a staircase approximation of the refractive index change (Fig. 8a), we calculated the equivalent $\Delta h$ as being $\Delta h \approx 7 \mathrm{~nm}$ as designated by the red point in Fig. 8b. In the following we apply this value of $\Delta h$ to model the effect of nonlinearity.

The correlation between the overlap $\eta$ and FOM for the two designs presented in Fig. 3a and $\mathrm{b}$ is shown in Fig. 9. Since the mode is concentrated in the chalcogenide slab, the overlap $\eta$ is larger for smaller height $Y$ and rapidly decreases for larger $Y$ (see Fig. 9a). Although the overlap of the modes is similar for the two designs, the higher losses of the design without the air gaps (see inset in Fig. 9a) leads to higher FOM. The structure without gaps has a 10 times larger FOM, which tends to saturate for larger waveguide heights. The saturation means that the decrease of attenuation fully compensates the decrease of overlap: when the optical mode is quite far from the slit, the losses and overlap are simultaneously reduced.
Dependence of the FOM and overlap $\eta$ on the slit width is presented in Fig. 9b. The overlap coefficient $\eta$ is smaller for the smaller widths $w$ in the design without air gaps, because the field enhancement cannot compensate the rapid decrease of the terahertz field. Since $L_{\pi / 2}$ stays approximately the same for the fixed $\Delta n$ and $\Delta h$, the FOM decreases mainly because of reduction of losses as the optical mode gets farther from the slit edges. However, for larger $w$, the terahertz field enhancement $G$ is reduced and, therefore, it can be more difficult to reach the required refractive index change $\Delta n=0.001$. So, a wider slit width does not necessarily result in an improved FOM. The conclusion of the FOM analysis is that the design shown in Fig. 3a is beneficial due to much smaller losses.

The necessary intensity of the incident $\mathrm{THz}$ field can be estimated from the Kerr type of nonlinearity,

$I_{i n c}=\frac{\Delta n}{n_{2} G^{2}}$,

while the expression for the incident $\mathrm{THz}$ field takes the form

$\left|E_{\text {inc }}^{T H z}\right|=\frac{1}{G} \sqrt{\frac{2 \Delta n}{n_{2} n_{0}^{T H z} \varepsilon_{0} c}}$

The nonlinear refractive index $n_{2}$ in the THz range is assumed to have the similar value as in optics [31]. Taking $n_{2}=1.1 \times 10^{-17} \mathrm{~m}^{2} / \mathrm{W}$ for chalcogenide glass $\mathrm{As}_{2} \mathrm{~S}_{3}$ [34], $\Delta n=0.001, n_{0}^{T H z}=2.7$, and field enhancement $G=100$ we estimate intensity $I_{i n c}=9 \times 10^{9} \mathrm{~W} / \mathrm{m}^{2}$ and field $\left|E_{i n c}^{T H z}\right|=16 \mathrm{kV} / \mathrm{cm}$. In fact, in order to make 

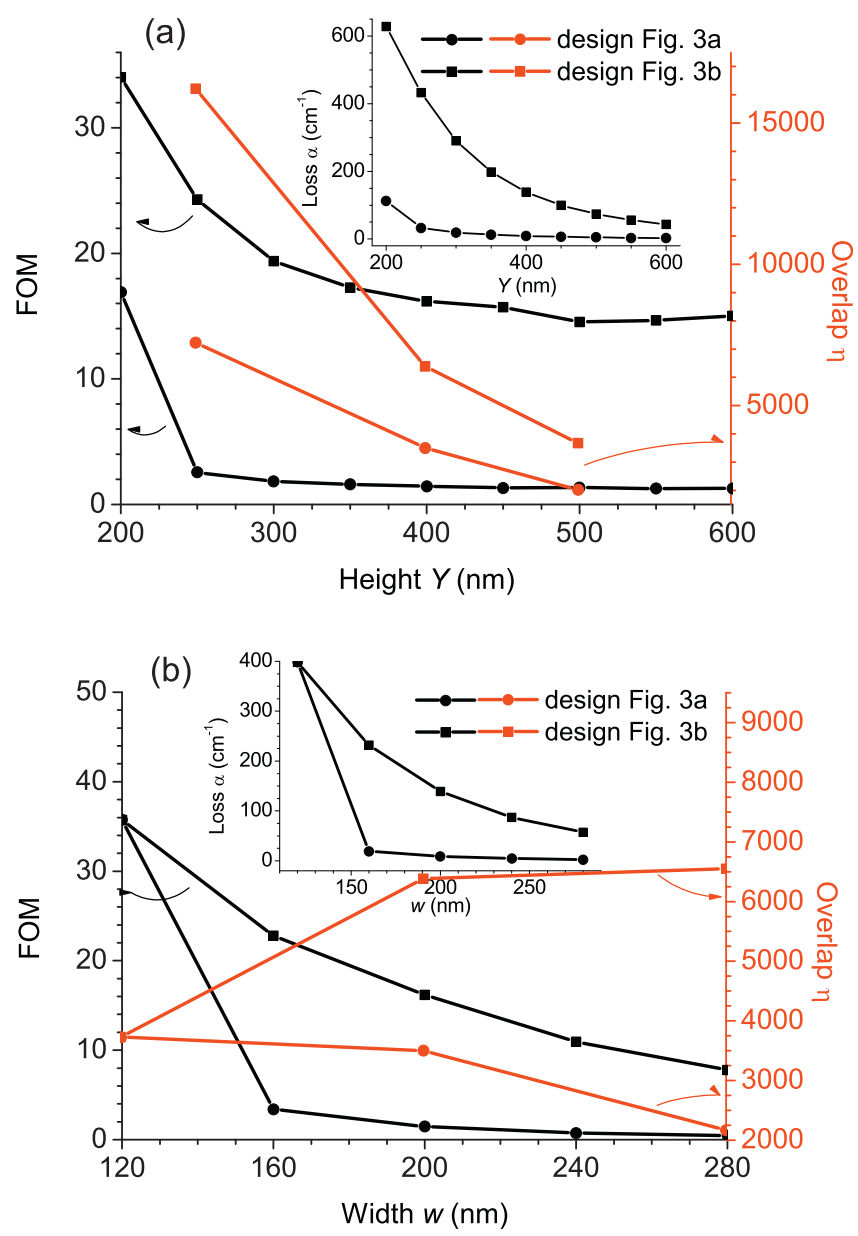

Fig. 9. Comparison of FOM and overlap for two designs as function of (a) waveguide height $Y$ and (b) slit width $w$. Position of the circular dot relates to both left- and right-hand scale. Losses are shown in the insets. Parameters: $Y=400 \mathrm{~nm}, X=120 \mathrm{~nm}, h=100 \mathrm{~nm}, w=200 \mathrm{~nm}, \lambda_{\text {opt }}=700 \mathrm{~nm}$.

practical use of the THz-induced phase change, a full $\pi /$ 2 phase change is not required. Thus, the requirements for the field intensities are softened, and the device would be useful in applications even with sub-kV/cm $\mathrm{THz}$ field strengths. We note that if a high enough field enhancement factor can be reached, one can, in principle, use silica as the nonlinear waveguide material. In the case of $G=100$, considering $n_{2}=3.2 \times 10^{-20} \mathrm{~m}^{2} / \mathrm{W}$ we obtain $I_{\text {inc }}=3 \times 10^{12} \mathrm{~W} /$ $\mathrm{m}^{2}$ [34] and a field strength of $\left|E_{i n c}^{T H z}\right|=295 \mathrm{kV} / \mathrm{cm}$, values that can be reached in tabletop laboratory environments with femtosecond amplified laser systems, but hardly at repetition rates relevant for highspeed optical communication systems (see $[53,54]$ ).

If we induce anisotropy and the optical field possesses either $x$ or $y$ polarization, then the nonlinear response is different in $x$ and $y$ directions. As the consequence the mode propagates with different nonlinear refractive indices $n_{2}^{x}$ and $n_{2}^{y}$. The results of all simulations presented in the paper are valid, given that the relevant scalar quantities are replaced by their vectorial counterparts (e.g. for the $y$-polarized optical wave $n_{2}$ should be replaced by $n_{2}^{y}$ ).

\section{Conclusion}

We have analyzed three different designs to impose optical wave control with a $\mathrm{THz}$ field. By making the justified assumption that nonlinearities in the $\mathrm{THz}$ range are of the same order of magnitude as in the optical range we utilize the non-resonant effect of $\mathrm{THz}$ field enhancement in nanoslits to achieve high local fields and thus high nonlinearities. Two feasible designs are studied in detail. The first is designed to have low losses (long propagation distance) and ensure a large phase shift. The second design is more favorable from the fabrication point of view, but suffers from higher modal losses. We have studied both the $\mathrm{THz}$ and optical field 
distributions for the different designs, and introduced two parameters that allowed us to quantify the performance of the structures. The first one is the coefficient of the overlap of optical and terahertz fields, and the second is the figure of merit defined as the ratio of the propagation length required to attain the $\pi / 2$ phase change to the propagation length of the optical mode. The FOM analysis indicates that the design with lower losses is preferable from a pure performance point of view. Evaluation of the strengths of the required terahertz fields has shown that they are quite reasonable for both highly nonlinear materials (exemplified here by chalcogenide glass) and, provided high enough field enhancement, even for ordinary glasses like silica.

\section{References}

[1] P.H. Siegel, Terahertz technology, IEEE Transactions on Microwave Theory and Techniques 50 (2002) 910-928.

[2] M. Tonouchi, Cutting-edge terahertz technology, Nature Photonics 1 (2007) 97-105.

[3] J. Federici, L. Moeller, Review of terahertz and subterahertz wireless communications, Journal of Applied Physics 107 (2010) 111101.

[4] K. Tanaka, H. Hirori, M. Nagai, THz nonlinear spectroscopy of solids, IEEE Transactions on Terahertz Science and Technology 1 (2011) 301-312.

[5] P.U. Jepsen, D.G. Cooke, M. Koch, Terahertz spectroscopy and imaging - modern techniques and applications, Laser Photonics Reviews 5 (2011) 124-166.

[6] T.J. Yen, W.J. Padilla, N. Fang, D.C. Vier, D.R. Smith, J.B Pendry, D.N. Basov, X. Zhang, Terahertz magnetic response from artificial materials, Science 303 (2004) 1494-1496.

[7] M. Choi, S.H. Lee, Y. Kim, S.B. Kang, J. Shin, M.H. Kwak, K.Y. Kang, Y.-H. Lee, N. Park, B. Min, A terahertz metamaterial with unnaturally high refractive index, Nature 470 (2011) 369374.

[8] L. Ju, B. Geng, J. Horng, C. Girit, M. Martin, Z. Hao, H.A. Bechtel, X. Liang, A. Zettl, Y.R. Shen, F. Wang, Graphene plasmonics for tunable terahertz metamaterials, Nature Nanotechnology 6 (2011) 630-634.

[9] H.-T. Chen, H. Lu, A.K. Azad, R.D. Averitt, A.C. Gossard, S.A Trugman, J.F. O'Hara, A.J. Taylor, Electronic control of extraordinary terahertz transmission through subwavelength metal hole array, Optics Express 16 (2008) 7641-7648.

[10] H.-T. Chen, W.J. Padilla, M.J. Cich, A.K. Azad, R.D. Averitt, A.J. Taylor, A metamaterial solid-state terahertz phase modulator, Nature Photonics 3 (2009) 148-151.

[11] H.-T. Chen, H. Yang, R. Singh, J.F. O'Hara, A.K. Azad, S.A. Trugman, Q.X. Jia, A.J. Taylor, Tuning the resonance in hightemperature superconducting terahertz metamaterials, Physical Review Letters 105 (2010) 247402.

[12] E. Ekmekci, A.C. Strikwerda, K. Fan, G. Keiser, X. Zhang, G. Turhan-Sayan, R.D. Averitt, Frequency tunable terahertz metamaterials using broadside coupled split-ring resonators, Physical Review B 83 (2011) 193103.

[13] Q. Zhou, Y. Shi, A. Wang, L. Li, D. Zhao, J. Liu, H. Sun, C. Zhang, Ultrafast optical modulation of terahertz metamaterials, Journal of Optics 13 (2011) 125102.
[14] J. Hebling, M.C. Hoffmann, H.Y. Hwang, K.L. Yeh, K.A. Nelson, Observation of nonequilibrium carrier distribution in $\mathrm{Ge}, \mathrm{Si}$, and $\mathrm{GaAs}$ by terahertz pump-terahertz probe measurements, Physical Review B 81 (2010) 035201.

[15] M.C. Hoffmann, J. Hebling, H.Y. Hwang, K.L. Yeh, K.A. Nelson, Impact ionization in InSb probed by terahertz pumpterahertz probe spectroscopy, Physical Review B 79 (2009) 161201

[16] F. Blanchard, D. Golde, F.H. Su, L. Razzari, G. Sharma, R. Morandotti, T. Ozaki, M. Reid, M. Kira, S.W. Koch, F.A. Hegmann, Effective mass anisotropy of hot electrons in nonparabolic conduction bands of $\mathrm{n}$-doped $\mathrm{InGaAs}$ films using ultrafast terahertz pump-probe techniques, Physical Review Letters 107 (2011) 107401.

[17] K. Shinokita, H. Hirori, M. Nagai, N. Satoh, Y. Kadoya, K. Tanaka, Dynamical Franz-Keldysh effect in GaAs/AlGaAs multiple quantum wells induced by single-cycle terahertz pulses, Applied Physical Letters 97 (2010) 211902.

[18] H. Hirori, M. Nagai, K. Tanaka, Excitonic interactions with intense terahertz pulses in $\mathrm{ZnSe} / \mathrm{ZnMgSSe}$ multiple quantum wells, Physical Review B 81 (2010) 081305.

[19] H. Hirori, K. Shinokita, M. Shirai, S. Tani, Y. Kadoya, K. Tanaka, Extraordinary carrier multiplication gated by a picosecond electric field pulse, Nature Communications 2 (2011) 594

[20] B. Zaks, R.B. Liu, M.S. Sherwin, Experimental observation of electron-hole recollisions, Nature 483 (2012) 580-583.

[21] D. Turchinovich, J.M. Hvam, M.C. Hoffmann, Self-phase modulation of a single-cycle terahertz pulse by nonlinear free-carrier response in a semiconductor, Physical Review B 85 (2012), 201304(R).

[22] M. Liu, H.Y. Hwang, H. Tao, A.C. Strikwerda, K. Fan, G.R. Keiser, A.J. Sternbach, K.G. West, S. Kittiwatanakul, J. Lu, S.A. Wolf, F.G. Omenetto, X. Zhang, K.A. Nelson, R.D. Averitt, Terahertz-field-induced insulator-to-metal transition in vanadium dioxide metamaterial, Nature 487 (2012) 345-348.

[23] M. Jewariya, M. Nagai, K. Tanaka, Ladder climbing on the anharmonic intermolecular potential in an amino acid microcrystal via an intense monocycle terahertz pulse, Physical Review Letters 105 (2010) 203003.

[24] A. Novitsky, M. Zalkovskij, R. Malureanu, P.U. Jepsen, A.V. Lavrinenko, Optical waveguide mode control by nanoslit-enhanced terahertz field, Optics Letters 37 (2012) 3903-3905.

[25] M.A. Seo, H.R. Park, S.M. Koo, D.J. Park, J.H. Kang, O.K. Suwal, S.S. Choi, P.C.M. Planken, G.S. Park, N.K. Park, Q.H. Park, D.S. Kim, Terahertz field enhancement by a metallic nano slit operating beyond the skin-depth limit, Nature Photonics 3 (2009) 152-156.

[26] H.J. Lezec, A. Degiron, E. Devaux, R.A. Linke, L. MartinMoreno, F.J. Garcia-Vidal, T.W. Ebbesen, Beaming light from a subwavelength aperture, Science 297 (2002) 820-822.

[27] J.H. Kang, D.S. Kim, Q-H. Park, Local capacitor model for plasmonic electric field enhancement, Physical Review Letters 102 (2009) 093906.

[28] Q.-H. Park, Optical antennas and plasmonics, Contemporary Physics 50 (2009) 407-423.

[29] J.W. Lee, T.H. Park, P. Nordlander, D.M. Mittleman, Terahertz transmission properties of an individual slit in a thin metallic plate, Optics Express 17 (2009) 12660-12667.

[30] A. Novitsky, M. Zalkovskij, R. Malureanu, A. Lavrinenko, Microscopic model of the $\mathrm{THz}$ field enhancement in a metal nanoslit, Optics Communications 284 (2011) 5495-5500. 
[31] M.C. Hoffmann, N.C. Brandt, H.Y. Hwang, K.-L. Yeh, K.A. Nelson, Terahertz Kerr effect, Applied Physics Letters 95 (2009) 231105.

[32] W.S. Rodney, I.H. Malitson, T.A. King, Refractive index of arsenic trisulfide, Journal of Optical Society of America 48 (1958) 633-636.

[33] A. Zakery, Low loss waveguides in pulsed laser deposited arsenic sulfide chalcogenide films, Journal of Physics D: Applied Physics 35 (2002) 2909-2913.

[34] B.J. Eggleton, B. Luther-Davies, K. Richardson, Chalcogenide photonics, Nature Photonics 5 (2011) 141-148.

[35] S.N. Taraskin, S.I. Simdyankin, S.R. Elliott, J.R. Neilson, T. Lo, Universal features of terahertz absorption in disordered materials, Physical Review Letters 97 (2006) 055504.

[36] E.P.J. Parrott, J.A. Zeitler, L.F. Gladden, S.N. Taraskin, S.R. Elliott, Extracting accurate optical parameters from glasses using terahertz time-domain spectroscopy, Journal of Non-Crystalline Solids 355 (2009) 1824-1827.

[37] M. Zalkovskij, C.Z. Bisgaard, A. Novitsky, R. Malureanu, D. Savastru, A. Popescu, P.U. Jepsen, A.V. Lavrinenko, Ultrabroadband terahertz spectroscopy of chalcogenide glasses, Applied Physics Letters 100 (2012) 031901.

[38] Q. Wu, X.C. Zhang, Free-space electro-optic sampling of terahertz beams, Applied Physics Letters 67 (1995) 3523-3525.

[39] P.U. Jepsen, C. Winnewisser, M. Schall, V. Schyja, S.R. Keiding, $\mathrm{H}$. Helm, Detection of THz pulses by phase retardation in lithium tantalate, Physical Review E 53 (1996) R3052-R3054.

[40] A. Nahata, D.H. Auston, T.F. Heinz, C.J. Wu, Coherent detection of freely propagating terahertz radiation by electro-optic sampling, Applied Physics Letters 68 (1996) 150-152.

[41] Z. Jiang, X.-C. Zhang, Electro-optic measurement of THz field pulses with a chirped optical beam, Applied Physics Letters 72 (1998) 1945-1947.

[42] K. Iwaszczuk, A. Andryieuski, A. Lavrinenko, X.C. Zhang, P.U. Jepsen, Non-invasive terahertz field imaging inside parallel plate waveguides, Applied Physics Letters 99 (2011) 071113

[43] X. Gai, T. Han, A. Prasad, S. Madden, D.-Y. Choi, R. Wang, D. Bulla, B. Luther-Davies, Progress in optical waveguides fabricated from chalcogenide glasses, Optics Express 18 (2010) 26635-26646.
[44] K. Suzuki, T. Baba, Nonlinear light propagation in chalcogenide photonic crystal slow light waveguides, Optics Express 18 (2010) 26675-26685.

[45] M. Galili, J. Xu, H.C.H. Mulvad, L.K. Oxenløwe, A.T. Clausen, P. Jeppesen, B. Luther-Davies, S. Madden, A. Rode, D.-Y. Choi, M. Pelusi, F. Luan, B.J. Eggleton, Breakthrough switching speed with an all-optical chalcogenide glass chip: $640 \mathrm{Gbit} / \mathrm{s}$ demultiplexing, Optics Express 17 (2009) 2182-2187.

[46] Z. Yang, M.K. Fah, K.A. Reynolds, J.D. Sexton, M.R. Riley, M.L. Anne, B. Bureau, P. Lucas, Opto-electrophoretic detection of bio-molecules using conducting chalcogenide glass sensors, Optics Express 18 (2010) 26754-26759.

[47] M. Kastner, D. Adler, H. Fritzsche, Valence-alternation model for localized gap states in lone-pair semiconductors, Physical Review Letters 37 (1976) 1504-1507.

[48] H. Xiang, S. Wang, Z. Wang, Z. Li, H. Yang, Q. Gong, X. Wang, X. Zhao, S. Gu, Laser irradiation induced enhancement on the ultrafast third-order optical nonlinearity of chalcogenide glass, Optical Materials 28 (2006) 1020-1024.

[49] O.N. Oliveira Jr., Langmuir-Blodgett films - properties and possible applications, Brazilian Journal of Physics 22 (1992) 60 69.

[50] K. Kamiyama, M. Era, T. Tsutsui, S. Saito, Third-harmonic generation in highly-oriented poly (2.5-dimethoxy-p-phenylevinylene) film prepared by use of the Langmuir-Blodgett technique, Japanese Journal of Applied Physics 29 (1990) L840L842.

[51] http://www.cst.com/.

[52] M. Naftaly, R.E. Miles, Terahertz beam interactions with amorphous materials, in: R.E. Miles, et al. (Eds.), Terahertz Frequency Detection and Identification of Materials and Objects, Springer, Dordrecht, 2007, pp. 107-122.

[53] H. Hirori, A. Doi, F. Blanchard, K. Tanaka, Single-cycle terahertz pulses with amplitudes exceeding $1 \mathrm{MV} / \mathrm{cm}$ generated by optical rectification in $\mathrm{LiNbO}_{3}$, Applied Physics Letters 98 (2011) 091106.

[54] K. Iwaszczuk, A. Andryieuski, A. Lavrinenko, X.C. Zhang, P.U. Jepsen, Terahertz field enhancement to the $\mathrm{MV} / \mathrm{cm}$ regime in a tapered parallel plate waveguide, Optics Express 20 (2012) 8344-8355. 